\title{
Learning Complex Representations from Spatial Phase Statistics of Natural Scenes
}

\author{
HaDi MaBouDi *广 \\ School of Cognitive Sciences \\ Institute for Research in Fundamental Sciences (IPM) \\ Tehran, Iran \\ h.d.maboudi@qmul.ac.uk \\ Hamid Soltanian-Zadeh \\ School of Cognitive Sciences \\ Institute for Research in Fundamental Sciences (IPM) \\ Tehran, Iran \\ hamids@rad.hfh.edu
}

\author{
Hideaki Shimazaki * \\ RIKEN Brain Science Institute \\ Wako, Saitama, Japan \\ shimazaki@brain.riken.jp
}

\author{
Shun-ichi Amari \\ RIKEN Brain Science Institute \\ Wako, Saitama, Japan \\ amari@brain.riken.jp
}

\begin{abstract}
Natural scenes contain higher-order statistical structures that can be encoded in their spatial phase information. Nevertheless, little progress has been made in modeling phase information of images in order to understand efficient representation of image phases in the brain. Based on recent findings of spatial phase structure in natural scenes, we introduce a generative model of the phase information in the visual systems according to the efficient coding hypothesis. In this model, we assume independent priors for the amplitude and phase of the coefficients, and model the phase using a non-uniform distribution, which extends existing models of independent component analysis for complex-valued signals. The parameters of the proposed model are then estimated under the maximum-likelihood principle. Using simulated data, we show that the proposed model outperforms conventional models with a uniform phase prior in blind source separation of complex-valued signals. We then apply the proposed model to natural scenes in the Fourier domain. The learning yields nonlinear features specified by a pair of similar Gabor-like filters in quadratic phase structure. These features predict properties of phase sensitive complex cells in the visual cortex, and indicate that the phase sensitive complex cells are essential for removing redundancy in natural scenes.
\end{abstract}

\section{Introduction}

One of the successful guiding principles to understand visual systems in the brain is the efficient coding hypothesis [3]. According to this hypothesis, organizations of a visual system are adapted to regularities in natural scenes that an animal encounters. The efficient coding hypothesis has successfully guided us to construct physiologically plausible statistical models of neurons in early visual cortices. However, most of the previous models extracted information contained only in amplitudes of an image in the Fourier domain, and were blind to its phase structure. Contrary to the

\footnotetext{
*Equal contribution

${ }^{\dagger}$ Current affiliation: School of Biological and Chemical sciences, Queen Mary University of London, E1 4NS, London, UK.

${ }^{\ddagger}$ Other affilications: School of Electrical and Computer Engineering, University of Tehran, Iran; Department of Radiology, Henry Ford Health System, Detroit, MI, United States.
} 
assumption of the previous models, it is well known that the phase of an image contains significantly more perceptual information than the amplitude of an image [24]. Perceptually salient features such as edges and bars are encoded in the ventral visual cortex based on their phase congruency [2, 5, 7, 15]. It has also been shown that both simple and complex cells in macaque V1 are sensitive to the phase images [9, 16, 20, 28]. Nevertheless, constructing models of a visual system that utilizes the characteristic phase information in the natural scenes remains to be a challenging problem.

The classical linear generative models represent the natural images by linearly combining features (i.e., receptive fields), and by weighting them using different coefficients [22, 4]. The coefficients of the features (i.e., responses of the receptive fields) are learned from natural images so that they become as independent as possible, according to the efficient coding hypothesis. Nevertheless it is known that their dependency cannot completely removed. It was pointed out that the residual dependency in the responses of the receptive fields is conveniently described by using scalar and circular components [30, 21, 19]. This suggests to use complex representation (a pair of real and imaginary features) of the natural images [21, 8, 17]. A successful complex representation model may explain why nearby simple cells in the primary visual cortex are phase quadratic [26, 13], and support psychophysical studies which suggested image phases may be detected by combining responses of simple cells possessing two odd and even symmetric receptive fields [26].

In this study, we present a linear generative model of complex representation for natural images using a superposition of complex features (a pair of features). While we consider independent priors for the amplitude and phase of the coefficients, our attention is particularly paied to the phase distribution. MaBouDi et al. previously demonstrated that local phases of natural scenes detected by Gabor filters are characterized by not only uniform but also non-uniform phase distributions [19]. Based on this knowledge, we model the phase distribution using a mixture of von Mises distributions, and provide inference algorithms under the maximum likelihood principle. We then demonstrate the utility and neurophysiological implications of this approach by both blind source separation of simulated data and analysis of natural scenes.

\section{Complex-valued independent component analysis}

Let $X^{\text {obs }}=\left(X^{1}, X^{2}, \ldots, X^{T}\right)$ be a collection of a complex-valued matrix that is a Fourier transform of image patches with size $N$ pixels. These $T$ patches were selected randomly from natural scenes. If we whiten the complex-valued data, we can assume that the samples, $X^{t}(t=1, \ldots, T)$, are mutually uncorrelated with zero mean.

We consider the following complex-valued generative model for these observations. In this model, a complex domain of natural patches, $X$, is generated from a superposition of unknown $N$ complex features, $A_{i}\left(\in C^{N \times 1}\right)$, namely $X=\sum_{i=1}^{N} s_{i} A_{i}$. Here $s_{i}$ is a complex coefficients given as $s_{i}=s_{i}^{R}+j s_{i}^{I}(j=\sqrt{-1})$, where $s_{i}^{R}$ and $s_{i}^{I}$ are real and imaginary components of the coefficient. This equation can be summarized as $X=A S$, where $A=\left[A_{1}, A_{2}, \ldots, A_{N}\right]$ is a mixing invertible matrix and $S=\left[s_{1}, s_{2}, \ldots, s_{N}\right]^{T}$ is a vector of complex coefficients. We assume that the samples are generated by the complex linear model using the complex coefficients that are sampled from an independent distributions of $S$, namely $p_{s}(S)=\prod_{i=1}^{N} p_{s_{i}}\left(s_{i}\right)$. This means, for each $X^{t}$, the coefficients $s^{t}$ are chosen independently, although the theory is applicable to the case in which $s^{t}$ and $s^{t^{\prime}}$ are dependent. Using the de-mixing matrix $W=A^{-1}$ (i.e., $S=W X$ ), the Jacobian of the above linear transformation is given as $\operatorname{det}(\bar{W})$, where $\bar{W}=\left[W^{R},-W^{I} ; W^{I}, W^{R}\right]$ with $W^{R}$ and $W^{I}$ being the real and imaginary component of $W$, respectively. Thus given $p_{s}(S)$, the probability density function of $X$ is obtained as

$$
p_{X}(X)=\operatorname{det}(\bar{W}) \prod_{i=1}^{N} p_{s_{i}}\left(s_{i}\right) .
$$

The complex independent component analysis (cICA) aims to infer the transform matrix $A$ (or $W$ ) and source signals $S$ under the assumption of their independence.

In this study, we propose to model each complex coefficient by polar coordinates, and impose independence between the amplitude and phase components. Namely, we rewrite the complex coefficients as $s_{i}=r_{i} e^{j \varphi_{i}}$, where $r_{i}=\left|s_{i}\right|$ and $\varphi_{i}=\arctan \frac{s_{i}^{I}}{s_{i}^{R}}$ are the amplitude and phase 
components of $s_{i}$, respectively. Then the probability density function of $s_{i}$ is

$$
p_{s_{i}}\left(s_{i}\right)=\frac{p\left(r_{i}, \varphi_{i}\right)}{r_{i}}=\frac{1}{r_{i}} p_{r_{i}}\left(r_{i}\right) p_{\varphi_{i}}\left(\varphi_{i}\right) .
$$

Throughout this paper, we assume that the amplitude distribution $p_{r_{i}}\left(r_{i}\right)$ follows the gamma distribution with the shape parameter being 2 ,

$$
p_{r_{i}}\left(r_{i} ; \beta_{i}\right)=\beta_{i}^{2} r_{i} e^{-\beta_{i} r_{i}},
$$

where $\beta_{i}>0$ is a scale parameter. This distribution resembles the amplitude distribution obtained from responses of complex Gabor filters to natural scenes [19], and imposes sparseness on the complex coefficients. We let the shape parameter be 2 because we found that the optimization algorithm to estimate the complex features under the maximum likelihood principle results in the algorithms proposed by previous studies (see below). While the previous studies utilized only amplitude information (a flat phase prior), our approach based on the maximum likelihood principle allows us to use different types of phase priors, and compare their performance. In the following sections, we derive optimization algorithms by considering two types of prior knowledge on the distributions of the phase components of the complex coefficients.

Circular complex-valued ICA (c-cICA) In this approach termed a circular cICA (c-cICA), we assume a uniform phase distribution, $p_{\varphi_{i}}\left(\varphi_{i}\right)=1 / 2 \pi$. The goal is to estimate the linear transformation $W$ such that the elements of the complex coefficient vector, $S$, are as independent as possible through an iterative optimization procedure. We estimate the parameters of the c-cICA model under the maximum likelihood principle. Given that the image patches are sampled independently, Eq.1 gives the log-likelihood function of the model parameters:

$$
l\left(W, \beta ; X^{\mathrm{obs}}\right)=\sum_{t=1}^{T} \sum_{i=1}^{N} \log p_{s_{i}}\left(W_{i} X^{t}\right)+T \log \operatorname{det} \overline{\mathrm{W}},
$$

where $W_{i}$ is the $i$-th row of $W$. By considering the prior knowledge of amplitude, Eq. 2, and a uniform phase distribution, we have

$$
l\left(W, \beta ; X^{\mathrm{obs}}\right)=\sum_{t=1}^{T} \sum_{i=1}^{N}\left[2 \log \beta_{i}-\beta_{i} r_{i}^{t}\right]-T N \log 2 \pi+T \log \operatorname{det} \overline{\mathrm{W}} .
$$

Note that this log-likelihood function generalizes the contrast function used in the complex Fast ICA [6]. Moreover since this model assumes a uniform phase distribution for the complex random variables, it is applicable for separation of circular complex random variables [14]. The maximum likelihood estimates (MLEs) of the model can be obtained by gradient descent algorithms, using the following gradients:

$$
\begin{aligned}
& \frac{\partial l(W, \beta)}{\partial \beta_{i}}=\sum_{t=1}^{T}\left(\frac{2}{\beta_{i}}-r_{i}^{t}\right), \\
& \frac{\partial l(W, \beta)}{\partial W_{m, n}}=T\left(W^{-H}\right)_{m, n}-\sum_{t=1}^{T} \frac{\beta_{m} x_{n}^{t} s_{m}^{t}{ }^{*}}{r_{m}^{t}},
\end{aligned}
$$

where the superscripts $*$ and $H$ denote the conjugate of complex coefficient, $s_{m}$, and Hermitian transpose of de-mixing matrix $W$, respectively. Note that $r_{i}$ and $s_{m}$, are calculated from $W$ and $X$.

Modified circular complex-valued ICA (mc-cICA) The c-cICA model does not use phase information that may be contained in the image patches. However, it was previously reported that higher-order statistics of natural scene is additionally encoded in non-uniform bimodal phase distributions [19]. In this section, we extend the complex ICA, and construct a model that utilizes the non-uniform phase distributions.

More specifically, we model the phase distribution by a mixture of uniform and von-Mises distributions:

$$
p_{\varphi_{i}}\left(\varphi_{i} ; \kappa_{i}, \lambda\right)=\lambda \operatorname{vM}\left(\varphi_{\mathrm{i}} ; \kappa_{\mathrm{i}}, 0\right)+\lambda \operatorname{vM}\left(\varphi_{\mathrm{i}} ; \kappa_{\mathrm{i}}, \pi\right)+(1-2 \lambda) \frac{1}{2 \pi} .
$$


Here $\operatorname{vM}\left(\varphi_{\mathrm{i}} ; \kappa_{\mathrm{i}}, 0\right)$ is a von-Mises distribution for a circular variable $\varphi_{i}$ with zero mean and a concentration parameter $\kappa_{i}$. We assume the zero mean because the peak phase location is redundant when the features are learned from the data. Further, given the observations of spatial phase distributions in natural scenes [19], we consider symmetric bimodal phase distributions with two peaks separated by $\pi$. For simplicity we further assume equal contributions from each component $(\lambda=1 / 3)$. Then the phase distribution that can cover a uniform and a spectrum of bimodal phase distributions is simplified as

$$
p_{\varphi_{i}}\left(\varphi_{i} ; \kappa_{i}\right)=\frac{1}{3 \pi I_{0}\left(\kappa_{i}\right)} \cosh \left(\kappa_{i} \cos \varphi_{i}\right)+\frac{1}{6 \pi},
$$

where $I_{0}($.$) is the Bessel function of order 0$. This model is a modification of the previous circular cICA. We call this new model the modified circular cICA (mc-cICA).

The log-likelihood function of the mc-cICA model is

$$
l\left(\theta ; X^{\mathrm{obs}}\right)=\sum_{t=1}^{T} \sum_{i=1}^{N}\left[2 \log \beta_{i}-\beta_{i} r_{i}^{t}+\log \cosh \left(\kappa_{i} \cos \varphi_{i}^{t}\right)-\log 3 \pi I_{0}\left(\kappa_{i}\right)\right]+T \log \operatorname{det} \bar{W},
$$

where $\theta=\left(W,\left\{\beta_{i}, \kappa_{i}\right\}_{i=1}^{N}\right)$ is a vector of the model parameters.

The MLEs of the parameters in the proposed model are obtained by gradient descent algorithms using Eq. 6 and the following gradients:

$$
\begin{aligned}
\frac{\partial l(\theta)}{\partial \kappa_{i}} & =\sum_{t=1}^{T} \cos \varphi_{i}^{t} \tanh \left(\kappa_{i} \cos \varphi_{i}^{t}\right)-T \frac{I_{1}\left(\kappa_{i}\right)}{I_{0}\left(\kappa_{i}\right)} \\
\frac{\partial l(\theta)}{\partial W_{m, n}} & =T\left(W^{-H}\right)_{m, n}-\sum_{t=1}^{T}\left[\frac{\beta_{m} x_{n}^{t} s_{m}^{t}{ }^{*}}{2 r_{m}^{t}}+\frac{1}{2} j \kappa_{m} \sin \varphi_{i}^{t} \tanh \left(\kappa_{m} \cos \varphi_{m}^{t}\right) \frac{x_{n}^{t} s_{m}^{t}{ }^{*}}{r_{m}^{t}{ }^{2}}\right]
\end{aligned}
$$

where $I_{1}($.$) is the Bessel function of order 1. For both c-cICA and mc-cICA, we use the conjugate$ gradient method.

\section{Results}

Performance comparison using simulated data In this section, we evaluate efficiency of source separation by variants of the cICAs, using simulated data. For this goal, we generated a data set $X$ by mixing 10 independent complex-valued source signals $S$, using a random invertible matrix $A$. We considered two different types of the complex-valued source signals using the polar coordinates: In one data set, phases were sampled from a uniform distribution; in the other data set, phases were sampled from a bimodal distribution (Eq. 88). Amplitudes were sampled from Eq. 3 3 in both cases. In total, we generated 20 data sets for each case. The mixing matrix and parameters of the probability density function of sources were chosen randomly for each data set.

We then applied the various cICA models to estimate the de-mixing matrix $W$. We evaluated their performance as follows. If the source signals are perfectly separated by these algorithms, the product of the mixing matrix, $A$, and the estimated transformation $W$ must be close to a permutation of the identity matrix. This matrix $P=W A$ is called a performance matrix. Examples of the performance matrices $P$ obtained from the c-cICA and mc-cICA models are shown in Fig. 11. The performance matrix of the mc-cICA model is closer to the permutation of identity matrix than the performance matrix of the c-cICA model, which indicates that the mc-cICA performs better in source separation. The quality of separation is summarized by the Amari index [1] defined on this performance matrix as

$$
\mathrm{AI}=\sum_{\mathrm{m}=1}^{\mathrm{N}}\left(\sum_{\mathrm{n}=1}^{\mathrm{N}} \frac{\left|\mathrm{P}_{\mathrm{m}, \mathrm{n}}\right|}{\max _{\mathrm{k}}\left|\mathrm{P}_{\mathrm{m}, \mathrm{k}}\right|}-1\right)+\sum_{\mathrm{n}=1}^{\mathrm{N}}\left(\sum_{\mathrm{m}=1}^{\mathrm{N}} \frac{\left|\mathrm{P}_{\mathrm{m}, \mathrm{n}}\right|}{\max _{\mathrm{k}}\left|\mathrm{P}_{\mathrm{k}, \mathrm{n}}\right|}-1\right),
$$

where $P_{m, n}$ is a $(\mathrm{m}, \mathrm{n})$-element of $P$.

Using the Amari index, we compared the mc-cICA with c-cICA as well as the previously suggested complex FastICA algorithm [6]. Figure 2 shows performance of the models for separating mixed independent complex-valued signals. We computed mean and standard deviation of the Amari index for 20 data sets composed of different sample sizes. Overall the performance of all 3 models increases 

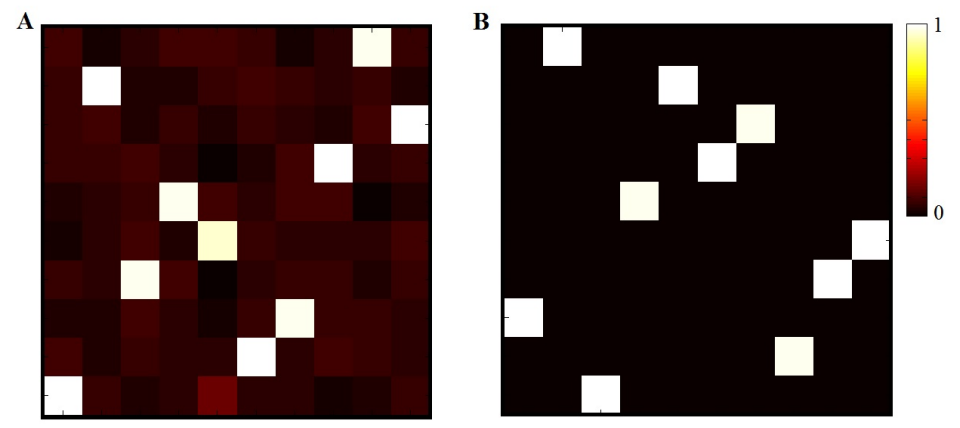

Figure 1: Examples of performance matrices obtained from c-cICA and mc-cICA. The performance matrix was computed as a product of a mixing matrix and a de-mixing matrix estimated by the two proposed algorithms (A: c-cICA, B: mc-cICA). The data were generated from a mixures of 10 independent complex-valued signals, using a non-uniform phase distribution. The two performance matrices are normalized by the absolute maximum value in each column. This comparison shows that the mc-cICA performs better than the c-cICA.
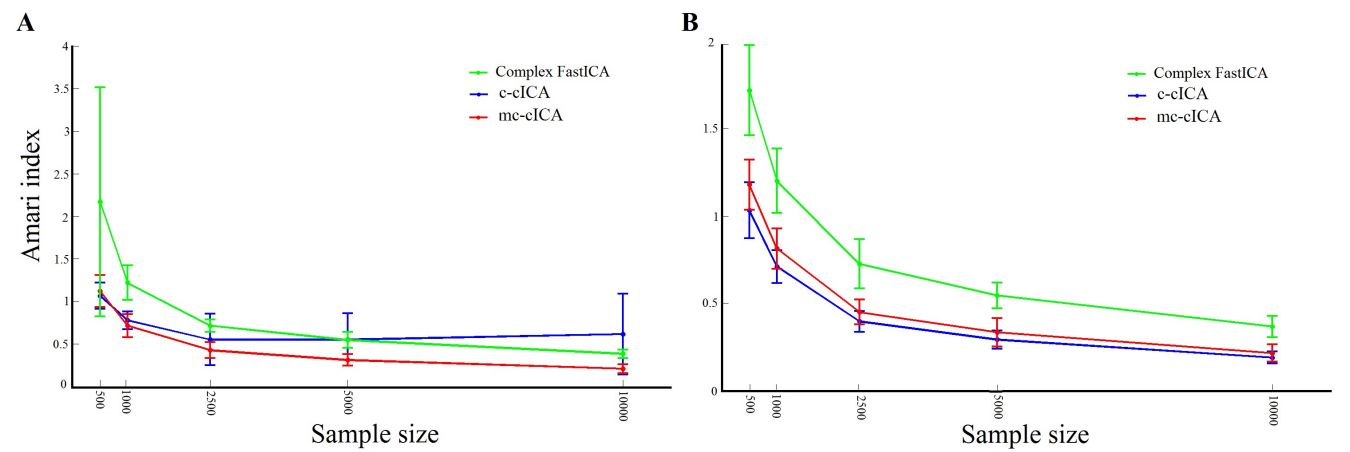

Figure 2: Comparison of model's performance in complex-valued signal separation. The Amari indexes of the models are plotted as a function of the sample size (a mean of 20 data sets $\pm \mathrm{SD}$ ). The data sets were constructed using a combination of a uniform and bimodal phase distributions (Left) or a uniform phase distribution (Right).

with the sample sizes. Figure 2 A shows the performance of models when they are applied to mixed complex signals generated by a combination of the bimodal and uniform phase distributions. Overall the mc-cICA outperformed the c-cICA and the complex Fast ICA for these data sets, although the performance of mc- and c-cICA were close in particular for small sample size. Figure $2 \mathrm{~B}$ exhibits performance of the models when they are applied to mixed complex signals generated by the uniform phase distribution (i.e., circular complex variables). For these data sets, the c-cICA and mc-cICA outperform the complex Fast ICA. Importantly, the performance of the mc-cICA approaches that of c-cICA whose assumption coincides with the data, indicating that the mc-cICA can successfully estimate the uniform phase distribution in the data.

Application of the mc-cICA to natural scenes In this section, we apply the mc-cICA model to natural scenes, and then analyze the optimal parameters of complex features learned from the natural scenes. We used the Hans van Hateren's repository of natural scenes [29] provided by Olshausen and Field [23]. We randomly selected 100,000 image patches with size $16 \times 16$ pixels from the natural scenes. We then computed the Fourier transform of each patch, and obtained the complex representation of natural scenes. After the DC components of each complex-valued patches were subtracted, we performed the complex whitening algorithm on the data. Finally, we applied the mc-cICA to the whitened natural patches to obtain the complex-valued features and source signals. 
bioRxiv preprint doi: https://doi.org/10.1101/112813; this version posted March 1, 2017. The copyright holder for this preprint (which was not certified by peer review) is the author/funder, who has granted bioRxiv a license to display the preprint in perpetuity. It is made available under aCC-BY 4.0 International license.

A
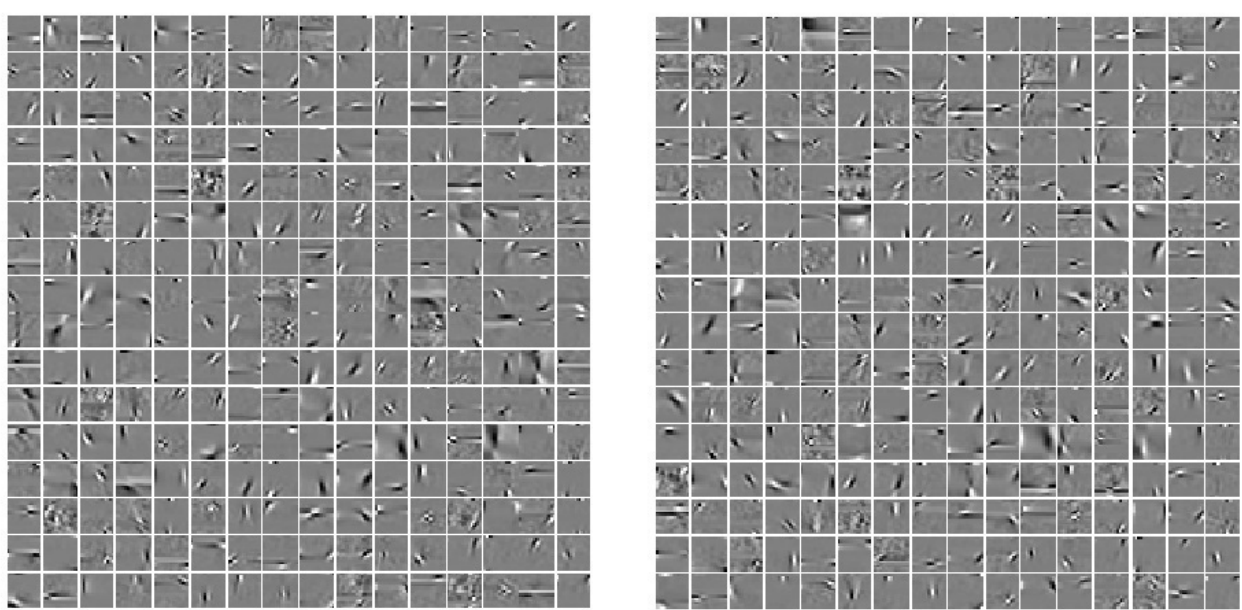

B
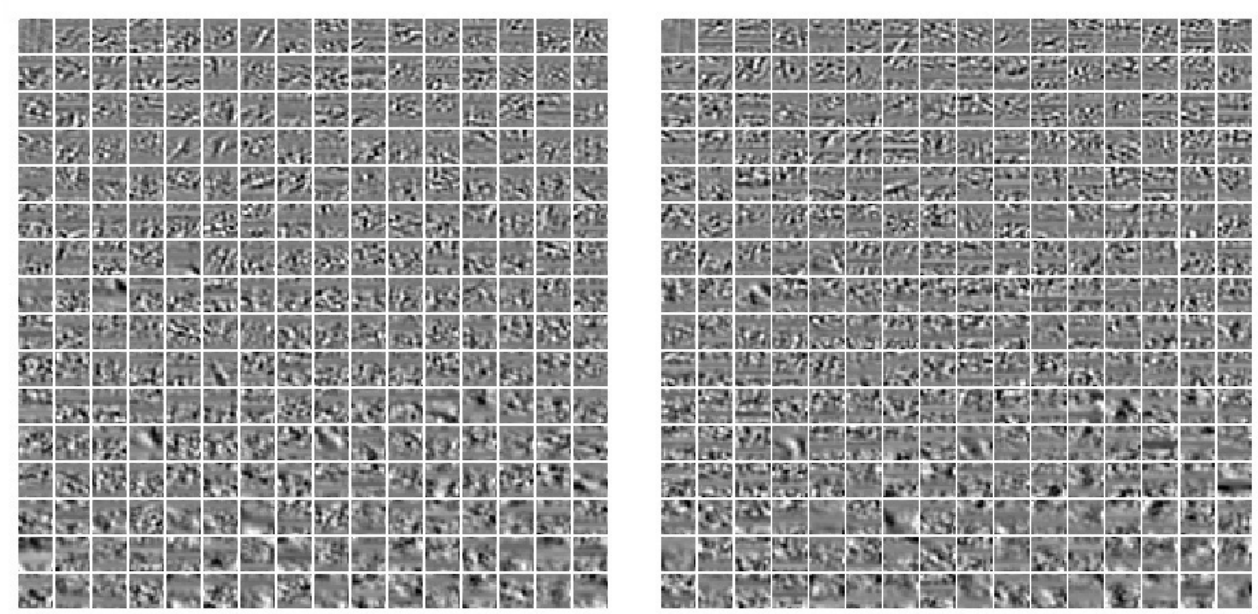

Figure 3: Complex features learned from natural scenes. The whole set of real (Left) and imaginary (Right) components of complex features obtained by the mc-cICA (panel A) and complex Fast ICA (panel B).

Figure $3 \mathrm{~A}$ shows the features extracted from the natural scenes in the original domain, i.e., an inverse transformation of the obtained complex-valued features to a real domain by multiplying them by the de-whitened matrix and inverse Fourier transform matrix. As a comparison, we show in Fig. $3 \mathrm{~B}$ features obtained from the complex Fast ICA applied to the same natural patches. By comparing the complex features obtained from the mc-cICA and complex Fast ICA (Figs $3 \mathrm{~A}$ and $3 \mathrm{~B}$ ), we conclude that the mc-cICA model provides the features that are more close to the receptive fields of neurons in early visual cortex than the complex FastICA model.

We further analyzed the learned complex features by comparing them with neurophysiological properties of V1 simple and complex cells. In order to quantify the learned features, we projected them to a space of parametric model of a simple cell receptive field: a two-dimensional Gabor function with five parameters that control location, frequency, phase and orientation of the filter. This artificial receptive field covers various selectivity of simple cells in the primary visual cortex [10, 25]. We extracted these parameters by changing the set of parameters of the Gabor-like receptive field, and finding the one that maximizes an inner product of the complex features with it.

The distribution of extracted frequency bandwidths shown in Fig. 44 covered the spatial frequency bandwidth of simple cells from 0.4 to 2.6 octaves [25, 10]. The median value of the learned complex 
A

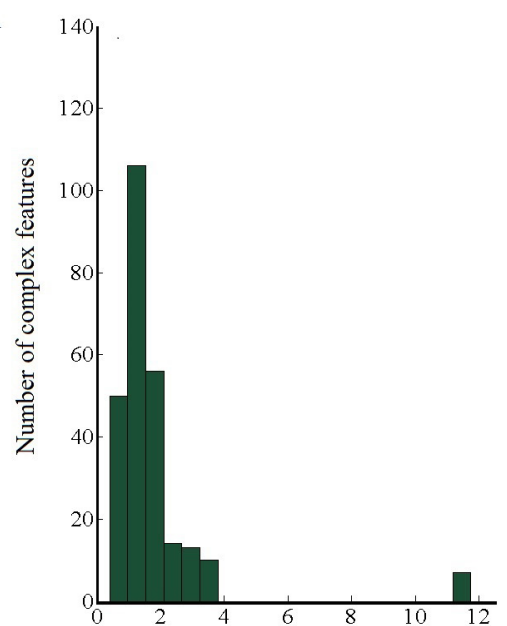

Spatial frequency bandwidth of

B

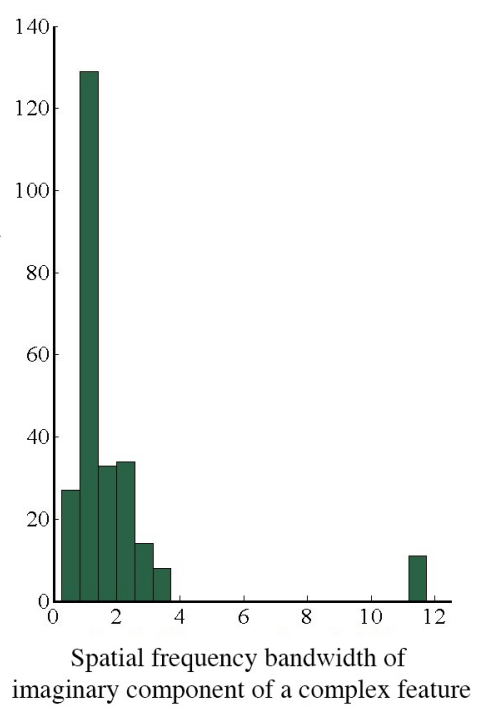

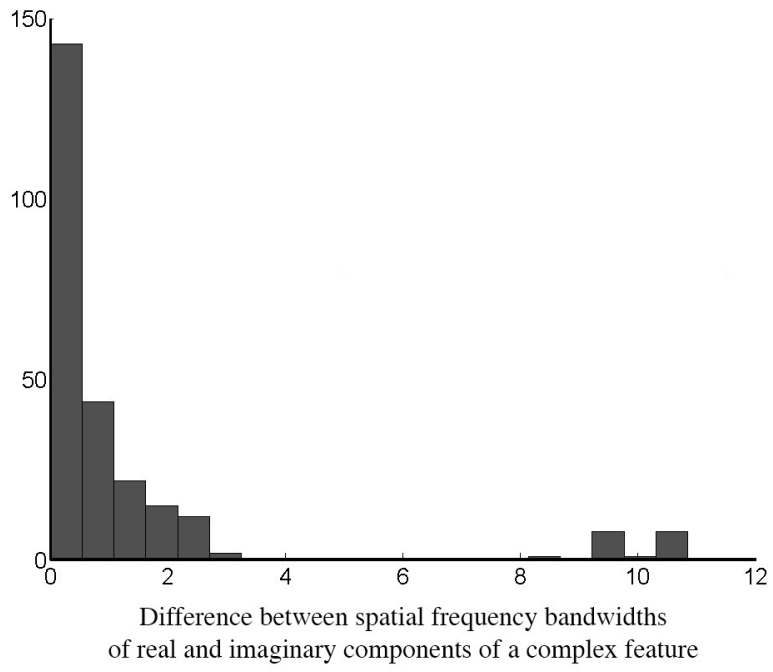

Figure 4: Histogram of spatial frequency bandwidths. (A) Histogram of extracted frequency bandwidths (octave) for real (Left) and imaginary (Right) components of learned complex features. A median for real and imaginary components is 1.318. (B) Histogram of difference between spatial frequency bandwidths of real and imaginary components. The real and imaginary components mostly exhibit the same frequency bandwidth.

features (1.318) was close to 1.4 octaves that was reported as a median value of macaque V1 neurons [27]. A histogram of difference between the extracted spatial frequency bandwidths of the real and imaginary components of the complex features (Fig. $4 \mathrm{~B}$ ) indicates that they exhibit mostly the same structure.

Figure 5.A displays a scatter plot of the spatial frequency bandwidth v.s. orientation bandwidth extracted from the real component of complex features. The orientation bandwidths were distributed uniformly irrespective of the spatial frequency bandwidths [12]. Further, the real and imaginary complex features were tuned to the same specific orientation bandwidth (Fig. 5B). Finally, the real and imaginary components of the complex features were orthogonal to each other (Fig. $5 \mathrm{C}$ ).

In summary, the real and imaginary components of the complex features learned from natural scenes resembled the Gabor-like receptive fields observed in neurophysiological literature. The real and imaginary components of complex features exhibited the same structures in spatial frequency, 
A

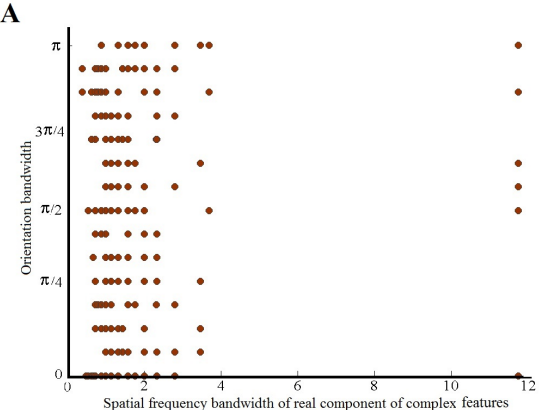

C

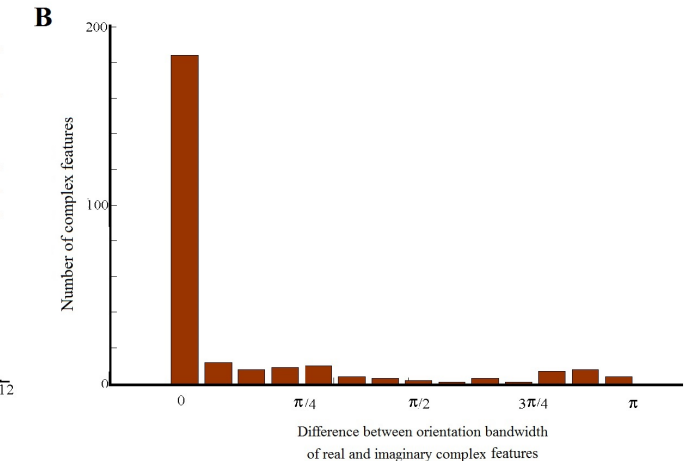

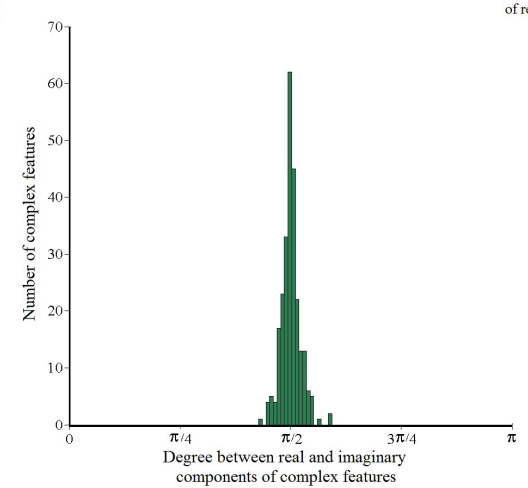

Figure 5: Orientation bandwidths and orthogonality of learned complex features. (A) Scatter plot of orientation bandwidth versus spatial frequency bandwidth of real components of complex features. (B) Histogram of differences between orientations of the real and imaginary components. (C) Orthogonality between the real and imaginary components of the complex features.

orientation and scale, but their phase are in quadrature (i.e., they are orthogonal), similarly to adjacent simple cells in early visual cortex [26, 13]. These features emerged even though we did not impose any assumption on their structure during training.

\section{Conclusion}

In this study, we suggested a generative model for complex representation of natural scenes in the visual cortex, which is applicable for separation of non-circular complex source signals. We demonstrated that, in blind source separation of complex-valued signals, the proposed model outperforms over and above the other methods that do not consider the phase information because the proposed model adaptively infers the underlying phase distribution including the uniform phase. Applied to natural scenes, we found that the components of learned complex feature better represent characteristic of the simple-cell receptive fields, and that the pair of components explains observed topographic relations between nearby simple cells. These results are consistent with proposals in signal processing to use quadrature pairs of Gabor filters [11, 18]. Under efficient coding hypothesis, these features suggest functions of phase sensitive complex cells in the redundancy reduction.

\section{References}

[1] S.-i. Amari, A. Cichocki, H. H. Yang, et al. A new learning algorithm for blind signal separation. Advances in neural information processing systems, pages 757-763, 1996.

[2] D. R. Badcock. How do we discriminate relative spatial phase? Vision Research, 24(12):1847-1857, 1984.

[3] H. B. Barlow. Possible principles underlying the transformations of sensory messages. 1961.

[4] A. J. Bell and T. J. Sejnowski. The "independent components" of natural scenes are edge filters. Vision research, 37(23):3327-3338, 1997. 
[5] P. J. Bennett and M. S. Banks. The effects of contrast, spatial scale, and orientation on foveal and peripheral phase discrimination. Vision research, 31(10):1759-1786, 1991.

[6] E. Bingham and A. Hyvärinen. A fast fixed-point algorithm for independent component analysis of complex valued signals. International journal of neural systems, 10(01):1-8, 2000.

[7] D. C. Burr, M. C. Morrone, and D. Spinelli. Evidence for edge and bar detectors in human vision. Vision research, 29(4):419-431, 1989.

[8] C. F. Cadieu and B. A. Olshausen. Learning intermediate-level representations of form and motion from natural movies. Neural computation, 24(4):827-866, 2012.

[9] N. A. Crowder, J. Van Kleef, B. Dreher, and M. R. Ibbotson. Complex cells increase their phase sensitivity at low contrasts and following adaptation. Journal of neurophysiology, 98(3):1155-1166, 2007.

[10] J. G. Daugman. Uncertainty relation for resolution in space, spatial frequency, and orientation optimized by two-dimensional visual cortical filters. JOSA A, 2(7):1160-1169, 1985.

[11] J. G. Daugman. Entropy reduction and decorrelation in visual coding by oriented neural receptive fields. Biomedical Engineering, IEEE Transactions on, 36(1):107-114, 1989.

[12] R. L. De Valois, E. W. Yund, and N. Hepler. The orientation and direction selectivity of cells in macaque visual cortex. Vision research, 22(5):531-544, 1982.

[13] G. C. DeAngelis, G. M. Ghose, I. Ohzawa, and R. D. Freeman. Functional micro-organization of primary visual cortex: receptive field analysis of nearby neurons. The Journal of Neuroscience, 19(10):4046-4064, 1999.

[14] J. Eriksson, A.-M. Seppola, and V. Koivunen. Complex ica for circular and non-circular sources. In Signal Processing Conference, 2005 13th European, pages 1-4. IEEE, 2005.

[15] D. J. Field and J. Nachmias. Phase reversal discrimination. Vision Research, 24(4):333-340, 1984.

[16] M. Hietanen, S. Cloherty, J. Van Kleef, C. Wang, B. Dreher, and M. Ibbotson. Phase sensitivity of complex cells in primary visual cortex. Neuroscience, 237:19-28, 2013.

[17] V. Laparra, M. U. Gutmann, J. Malo, and A. Hyvärinen. Complex-valued independent component analysis of natural images. In Artificial Neural Networks and Machine Learning-ICANN 2011, pages 213-220. Springer, 2011.

[18] T. S. Lee. Image representation using 2d gabor wavelets. Pattern Analysis and Machine Intelligence, IEEE Transactions on, 18(10):959-971, 1996.

[19] H. MaBouDi, H. Shimazaki, S.-i. Amari, and H. Soltanian-Zadeh. Representation of higher-order statistical structures in natural scenes via spatial phase distributions. Vision research, 2015.

[20] F. Mechler, D. S. Reich, and J. D. Victor. Detection and discrimination of relative spatial phase by v1 neurons. The Journal of neuroscience, 22(14):6129-6157, 2002.

[21] B. A. Olshausen, C. F. Cadieu, and D. K. Warland. Learning real and complex overcomplete representations from the statistics of natural images. In SPIE Optical Engineering + Applications, pages 74460S-74460S. International Society for Optics and Photonics, 2009.

[22] B. A. Olshausen et al. Emergence of simple-cell receptive field properties by learning a sparse code for natural images. Nature, 381(6583):607-609, 1996.

[23] B. A. Olshausen and D. J. Field. Sparse coding with an overcomplete basis set: A strategy employed by v1? Vision research, 37(23):3311-3325, 1997.

[24] A. V. Oppenheim and J. S. Lim. The importance of phase in signals. Proceedings of the IEEE, 69(5):529$541,1981$.

[25] N. Petkov and P. Kruizinga. Computational models of visual neurons specialised in the detection of periodic and aperiodic oriented visual stimuli: bar and grating cells. Biological cybernetics, 76(2):83-96, 1997.

[26] D. A. Pollen and S. F. Ronner. Phase relationships between adjacent simple cells in the visual cortex. Science, 212(4501):1409-1411, 1981. 
[27] B. C. Skottun, R. L. De Valois, D. H. Grosof, J. A. Movshon, D. G. Albrecht, and A. Bonds. Classifying simple and complex cells on the basis of response modulation. Vision research, 31(7):1078-1086, 1991.

[28] J. Touryan, G. Felsen, and Y. Dan. Spatial structure of complex cell receptive fields measured with natural images. Neuron, 45(5):781-791, 2005.

[29] J. H. van Hateren and A. van der Schaaf. Independent component filters of natural images compared with simple cells in primary visual cortex. Proceedings of the Royal Society of London B: Biological Sciences, 265(1394):359-366, 1998.

[30] B. Wegmann and C. Zetzsche. Statistical dependence between orientation filter outputs used in a humanvision-based image code. In Lausanne-DL tentative, pages 909-923. International Society for Optics and Photonics, 1990. 